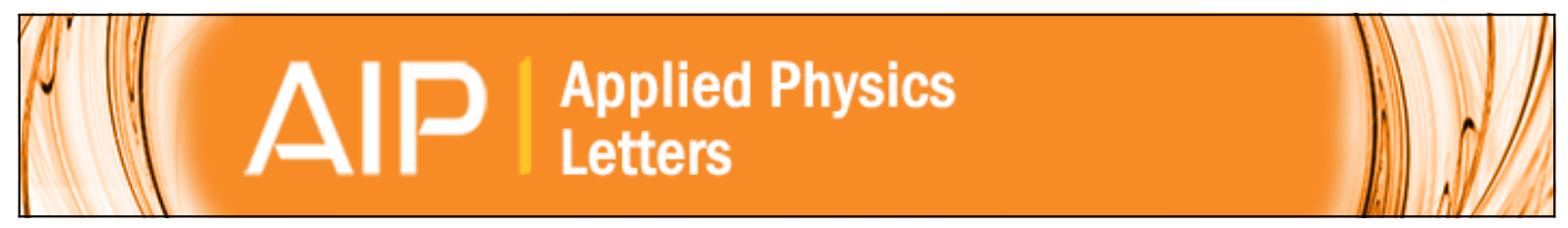

The environment of graphene probed by electrostatic force microscopy

J. Moser, A. Verdaguer, D. Jiménez, A. Barreiro, and A. Bachtold

Citation: Applied Physics Letters 92, 123507 (2008); doi: 10.1063/1.2898501

View online: http://dx.doi.org/10.1063/1.2898501

View Table of Contents: http://scitation.aip.org/content/aip/journal/apl/92/12?ver=pdfcov

Published by the AIP Publishing

\title{
AlP
}

\section{Create a profile.




\title{
The environment of graphene probed by electrostatic force microscopy
}

\author{
J. Moser, ${ }^{1, a)}$ A. Verdaguer, ${ }^{2}$ D. Jiménez, ${ }^{3}$ A. Barreiro, ${ }^{1}$ and A. Bachtold ${ }^{1, b)}$ \\ ${ }^{1}$ CIN2 amd CNM Barcelona, Campus UAB, E-08193 Bellaterra, Spain \\ ${ }^{2}$ ICN,Campus UAB, E-08193 Bellaterra, Spain \\ ${ }^{3}$ Departament d'Enginyeria Electrònica, Escola Tècnica Superior d'Enginyeria, Universitat Autònoma de \\ Barcelona, E-08193 Bellaterra, Spain
}

(Received 14 January 2008; accepted 26 February 2008; published online 26 March 2008)

\begin{abstract}
We employ electrostatic force microscopy to study the electrostatic environment of graphene sheets prepared with the micromechanical exfoliation technique. We detect the electric dipole of residues left from the adhesive tape during graphene preparation, as well as the dipole of water molecules adsorbed on top of graphene. Water molecules form a dipole layer that can generate an electric field as large as $\sim 10^{9} \mathrm{~V} \mathrm{~m}^{-1}$. We expect that water molecules can significantly modify the electrical properties of graphene devices. (C) 2008 American Institute of Physics. [DOI: 10.1063/1.2898501]
\end{abstract}

Graphene, a two-dimensional crystal of carbon atoms arranged in a honeycomb lattice, is among the thinnest objects imaginable. ${ }^{1,2}$ The structural properties of graphene make it a system of choice to study the physics of Dirac fermions; ${ }^{3,4}$ it is also envisioned as a building block for a novel generation of electronic devices. One inherent technological difficulty, however, remains: because all the atoms are at the surface and are directly exposed to the environment, the electronic properties are easily affected by unwanted adsorbates.

In this letter, we employ electrostatic force microscopy (EFM) to probe the electrostatic environment of graphene sheets. Two adsorbate species possessing an electric dipole are identified: water molecules and residues left from the adhesive tape during fabrication. Water molecules form a dipole layer on top of graphene that can generate an electric field as large as $\sim 10^{9} \mathrm{~V} \mathrm{~m}^{-1}$. Tape residues form an ultrathin layer nearby the graphene sheets on top of the silicon oxide substrate, which is not detectable using standard topographic atomic force microscopy (AFM).

We start by briefly describing our fabrication technique and our experimental setup. Graphene sheets are obtained using the conventional micromechanical exfoliation technique: ${ }^{5,6}$ a flake of bulk Kish graphite is repeatedly cleaved with an adhesive tape and pressed down onto a silicon wafer coated with $280 \mathrm{~nm}$ of thermal silicon oxide. Two types of adhesive tape are used: standard wafer protection tape for microfabrication by ICROS and Magic Tape by $3 \mathrm{M}$. Thin graphene sheets are optically located, imaged by AFM, and occasionally examined by Raman spectroscopy to identify single layer specimens. EFM measurements are carried out under various humidity conditions in a constant flow of either dry $\mathrm{N}_{2}$ gas, or moist $\mathrm{N}_{2}$ gas produced by bubbling dry $\mathrm{N}_{2}$ in de-ionized water.

The EFM technique ${ }^{7}$ is well suited to study dipoles on surfaces. Our EFM protocol proceeds in two passes along a given scan line: first, the topography is recorded in a tapping mode [Figs. 1(a) and 1(b)], then the AFM tip is lifted by a given amount, and a bias $V_{\mathrm{dc}}$ is applied to the Si backgate and a potential $V_{\mathrm{ac}} \cos (\omega t)$ is applied to the tip. The tip experiences a force whose term at the frequency $\omega$ reads [Fig. $1(\mathrm{c})]$

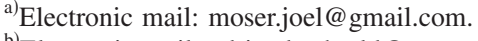

${ }^{b)}$ Electronic mail: adrian.bachtold@cnm.es.
}

$$
F_{\omega}=\frac{\partial C}{\partial z}\left(V_{\mathrm{dc}}-\Delta \phi\right) V_{\mathrm{ac}},
$$

where $C$ and $\Delta \phi$ are the capacitance and the contact potential difference between the sample and the tip. We measure $F_{\omega}$ over a range of $V_{\text {dc }}$ [Fig. 2(a) and Figs. 3(a)-3(c)]. As in

(a)

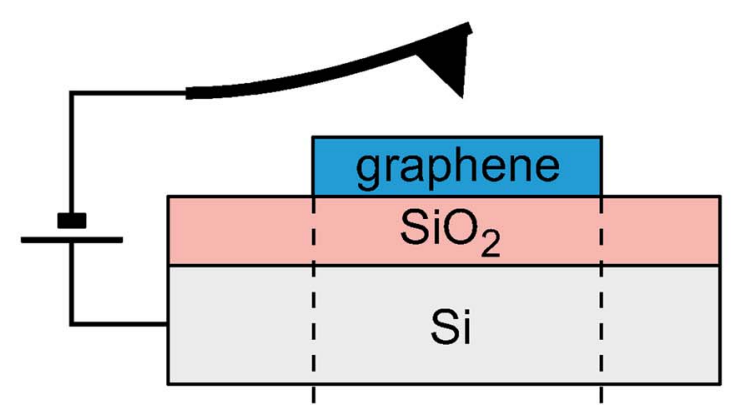

(b)

(c)
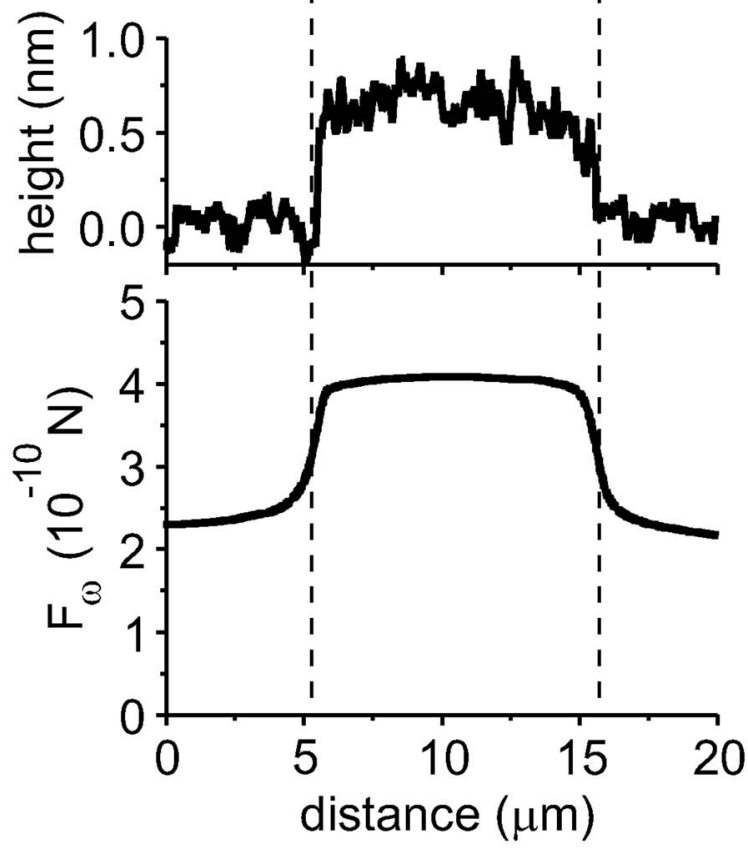

FIG. 1. (Color online) (a) Schematic of the EFM setup. (b) In a first pass, the topography of the graphene sheet is recorded. (c) In a second pass, a dc voltage $V_{\mathrm{dc}}$ is applied to the $\mathrm{Si}$ substrate and an ac voltage $V_{\mathrm{ac}}=1 \mathrm{mV}$ is applied to the tip at a frequency set to the resonance frequency of the cantilever. The sample is scanned at a constant height of about $50 \mathrm{~nm}$ and the oscillating force is measured. 

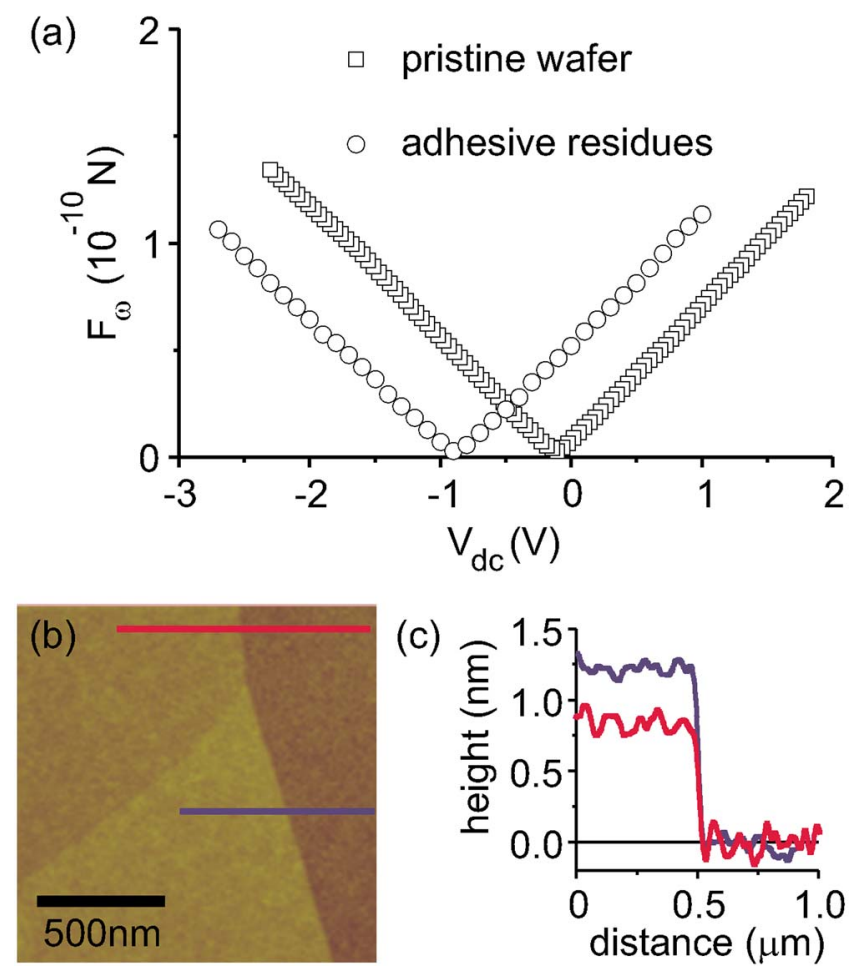

FIG. 2. (Color online) (a) Force term $F_{\omega}$ experienced by the AFM tip as a function of $V_{\mathrm{dc}}$ on a pristine $\mathrm{SiO}_{2}$ surface and on $\mathrm{SiO}_{2}$ covered with tape residues. [(b)-(c)] Topography of a typical graphene sheet. Plots in (c) are sections along the red and blue lines depicted in (b).

Kelvin probe force microscopy, $\Delta \phi$ is given by $V_{\mathrm{dc}}$ that minimizes $F_{\omega}$.

Prior to discussing the measurements, we recall the connection between $\Delta \phi$ and the presence of dipoles on surfaces. The energy $e \phi$ is the energy barrier that an electron has to overcome in order to be extracted from a material to the vacuum. Any electric field existing at the surface of this material, originating, e.g., from dipoles, modifies the contact potential $\phi$ by an amount $\chi: \phi=W+\chi$, where $W$ is the contact potential without electric field at the surface. For further insight into the underlying physics of $\Delta \phi$, see Refs. 8 and 9. In our experiment, the contact potential of the tip is $\phi_{\text {tip }}$, and the one of the Si wafer depends on whether the graphene sheet is present or not:

$$
\begin{aligned}
& \phi_{\mathrm{Si}}^{\text {graphene }}=W_{\mathrm{Si}}+\chi^{\text {graphene }}, \\
& \phi_{\mathrm{Si}}^{\text {no graphene }}=W_{\mathrm{Si}}+\chi^{\text {no graphene }} .
\end{aligned}
$$

We first look into the electrostatic traces left by the adhesive tape. In the case of a $\mathrm{SiO}_{2}$ surface onto which the adhesive tape has been pressed (without graphite), EFM measurements reveal that the contact potential difference $\Delta \phi=\phi_{\mathrm{Si}}^{\text {no graphene }}-\phi_{\text {tip }}$ is significantly shifted to negative values [Fig. 2(a)]. $\Delta \phi$ is found to vary between -2 and $-0.5 \mathrm{~V}$ depending on where on the wafer the measurement is carried out. This has to be compared with $\Delta \phi \simeq 0 \mathrm{~V}$, for a pristine wafer. Both adhesive tapes that we used yield similar results. This shift in $\Delta \phi$ suggests that adhesive residues change $\chi^{\text {no graphene }}$, which we attribute to the deposition of dipoles on the surface. Note that the shift in $\Delta \phi$ is stable over long periods of time, which suggests that it is not related to individual charges that would get neutralized, for example, by charged moiecules in the environment. (a)

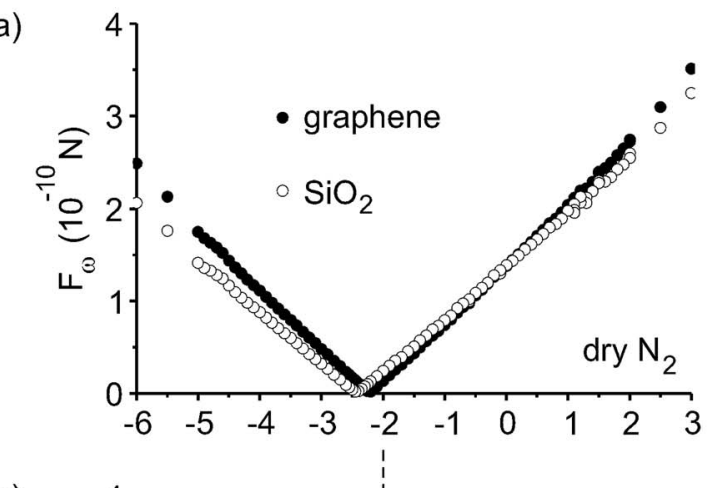

(b)
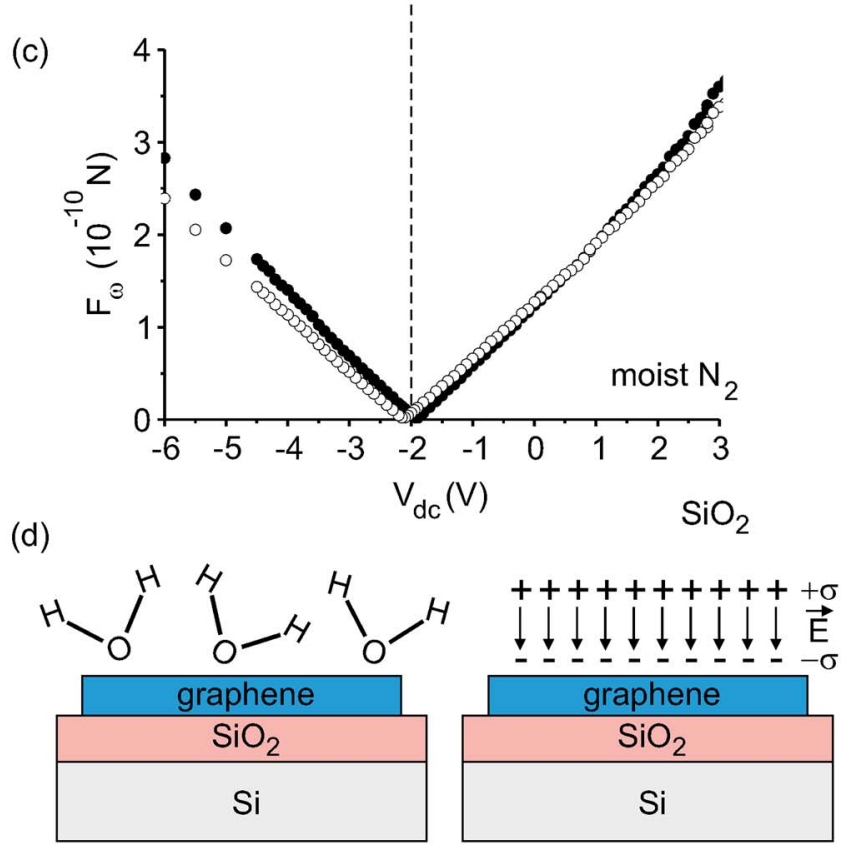

FIG. 3. (Color online) Force term $F_{\omega}$ experienced by the AFM tip as a function of $V_{\mathrm{dc}}$ in (a) dry $\mathrm{N}_{2}$; (b) dry $\mathrm{N}_{2}$ after heating the sample to $160{ }^{\circ} \mathrm{C}$ for $1 \mathrm{~h}$ in dry $\mathrm{N}_{2}$; (c) moist $\mathrm{N}_{2}$. Data on graphene and on silicon oxide $\sim 1 \mu \mathrm{m}$ away from graphene are shown. The dotted line at $V_{\mathrm{dc}}=-2 \mathrm{~V}$ draws the attention to the rather weak dependence on humidity of $F_{\omega}\left(V_{\mathrm{dc}}\right)$ measured on the oxide. (d) Water molecules adsorb on average with the oxygen atom pointing towards the graphene sheet, and form a dipole layer with an effective surface charge $\sigma$ (schematic not on scale).

These tape residues do not seem to roughen the surface, as inferred from topography measurements [Figs. 2(b) and 2(c)]. Presumably, this is why they have not been reported so far, as a standard topography scan fails to detect them. Further studies are needed, especially to find out whether or not these residues are present underneath the graphene sheets. At first sight, they should not, as the $\mathrm{SiO}_{2}$ surface is masked by the graphene sheets when pressing the tape down onto the wafer. However, micromechanical exfoliation is rather difficult to control, and residues may well lie beneath graphene sheets. The latter situation could have important conse- 
quences on the transport properties of graphene devices (see discussion below for water).

We now turn to EFM measurements on graphene sheets where we modify the humidity of the environment. Figure 3 compares measurements on a single graphene layer and on the oxide $\sim 1 \mu \mathrm{m}$ away. The measurement is first performed in dry $\mathrm{N}_{2}$ with a relative humidity of less than $3 \%$, thus corresponding to a submonolayer water coverage on clean $\mathrm{SiO}_{2}$ [Fig. 3(a)] (Ref. 10). Without taking it out of the dry environment, the sample is heated to $160{ }^{\circ} \mathrm{C}$ for $1 \mathrm{~h}$ and measured again [Fig. 3(b)]. Eventually, moist $\mathrm{N}_{2}$ is introduced until $\mathrm{RH} \simeq 50 \%\left[\sim 3\right.$ water monolayers on clean $\mathrm{SiO}_{2}$ (Ref. 10)] [see Fig. 3(c)]. $\Delta \phi$ on the graphene sheet is observed to significantly vary between each step $(-2.1 \mathrm{~V} \rightarrow$ $-0.7 \mathrm{~V} \rightarrow-1.9 \mathrm{~V}) .{ }^{11}$ By contrast, $\Delta \phi$ measured on the oxide stays pretty much constant $(\sim-2 \mathrm{~V})$, which suggests that $\phi_{\text {tip }}, W$, and $\chi^{\text {no graphene }}$ are not much affected by humidity. ${ }^{12}$ Therefore, the quantity that appears to be sensitive to water is $\chi^{\text {graphene }}$, which changes by $\sim 1.3 \mathrm{~V}$ from one step to another in Fig. 3. We attribute this strong variation to water molecules that desorb from and readsorb onto the graphene sheet. ${ }^{13}$

The adsorption of water on graphene may appear surprising, as it is well established that graphite is highly hydrophobic. ${ }^{14}$ However, it has been reported that water can adsorb on graphite for $\mathrm{RH} \simeq 60 \%$ at the surface defects such as steps. ${ }^{15}$ It has also been reported that water can adsorb on carbon structures such as carbon nanotubes ${ }^{16}$ and on selfassembled monolayers (SAM) of carbon chains. ${ }^{17}$ It has been argued that the key ingredient in water adsorption on SAM is the surface roughness. Interestingly, the surface of graphene is also corrugated as it tends to follow the roughness of the substrate. $^{18,19}$

Because $\Delta \phi=\phi_{\mathrm{Si}}^{\text {graphene }}-\phi_{\text {tip }}$ shifts to more negative values as humidity is raised, water molecules adsorb on average with the oxygen atoms pointing toward the graphene sheet. As such, the layer of water molecules can be described as a dipole layer with the negative charges toward the graphene sheet [see Fig. 3(d)]. The electric field is maximum within the dipole layer, and vanishes to zero as $e^{-a z}$ as the distance $z$ to the layer is increased (in the particular case where the layer consists of a periodic array of dipoles that are all pointing in the same direction, $a$ is the distance between two neighboring dipoles).

We can obtain a rough estimate for the strength of the electric field $E$ within the water dipole layer. Assuming that the width of the dipole layer is $d=1 \mathrm{~nm}$, we get $E$ $\simeq \Delta \chi^{\text {graphene }} / d \simeq 10^{9} \mathrm{~V} \mathrm{~m}^{-1}$, where $\Delta \chi^{\text {graphene }} \simeq 1 \mathrm{~V}$ is the shift in $\chi^{\text {graphene }}$ from dry to wet environment. As a comparison, we calculate $E$ to be of the order of $10^{9}-10^{10} \mathrm{~V} \mathrm{~m}^{-1}$ using the water electric dipole $6.2 \times 10^{-30} \mathrm{Cm}$, assuming a monolayer of water molecules that are all pointing in the direction perpendicular to the surface with a density from 1 to $10 \mathrm{~nm}^{-2}$, and modeling the dipole layer as a parallel plate capacitor. The strength of $E$ changes somewhat when considering that the dipoles are not all oriented in the same direction or can flip in the field.

An electric field of $10^{9} \mathrm{~V} \mathrm{~m}^{-1}$ within the dipole layer corresponds to the strength obtained when applying $\simeq 300 \mathrm{~V}$ between the Si backgate and a graphene device. It is important to note that the graphene sheet experiences a fraction of this field only, as it lies outside of the dipole layer. Nevertheiess, we expect that $E$ will significantly shift the Fermi level of graphene devices. ${ }^{20}$ Moreover, this field is likely to be inhomogeneous, resulting in puddles of electrons and holes near the charge neutrality point. ${ }^{21}$ Another consequence is that water can screen charge impurities ${ }^{22}$ as well as modify electron-electron interactions.

In conclusion, we show that EFM is a powerful tool for the characterization of the electrostatic environment of graphene. Water molecules form a dipole layer on top of graphene that generates a large electric field. We expect water to have a strong influence on the transport properties of graphene devices.

We are thankful to E. Hernández and F. Guinea for helpful discussions. We are indebted to F. Alsina for his help with the Raman characterization. This work was supported by an EURYI grant, FP6-IST-021285-2 and EXPLORA NAN2007-29375-E, Ministerio de Educación y Ciencia, Spain. D.J. acknowledges financial support from Ministerio de Educación y Ciencia under project TEC2006-13731C0201/MIC.

${ }^{1}$ A. Castro Neto, F. Guinea, and N. M. Peres, Phys. World 19(11), 33 (2006).

${ }^{2}$ A. K. Geim and K. S. Novoselov, Nat. Mater. 6, 183 (2007).

${ }^{3}$ K. S. Novoselov, A. K. Geim, S. V. Morozov, D. Jiang, M. I. Katsnelson, I. V. Grigorieva, S. V. Dubonos, and A. A. Firsov, Nature (London) 438, 197 (2005).

${ }^{4}$ Y. Zhang, Y.-W. Tan, H. L. Stormer, and P. Kim, Nature (London) 438 , 201 (2005).

${ }^{5}$ K. S. Novoselov, A. K. Geim, S. V. Morozov, D. Jiang, Y. Zhang, S. V. Dubonos, I. V. Grigorieva, and A. A. Firsov, Science 306, 666 (2004).

${ }^{6}$ K. S. Novoselov, D. Jiang, F. Schedin, T. J. Booth, V. V. Khotkevich, S. V. Morozov, and A. K. Geim, Proc. Natl. Acad. Sci. U.S.A. 102, 10451 (2005).

${ }^{7}$ U. Zerweck, C. Loppacher, T. Otto, S. Grafström, and L. M. Eng, Phys. Rev. B 71, 125424 (2005).

${ }^{8}$ J. Lü, E. Delamarche, L. Eng, R. Benewitz, E. Meyer, and H.-J. Güntherodt, Langmuir 15, 8184 (1999).

${ }^{9}$ N. W. Ashcroft and N. D. Mermin, Solid State Physics (Saunders College, Philadelphia, 1976), pp. 354-362.

${ }^{10}$ A. Verdaguer, C. Weis, G. Oncins, G. Ketteler, H. Bluhm, and M. Salmeron, Langmuir 23, 9699 (2007).

${ }^{11}$ The slope of $F_{\omega}\left(V_{\mathrm{dc}}\right)$ is different on the oxide and on the graphene sheet. This is attributed to the graphene sheet that changes the capacitance between the tip and the $\mathrm{Si}$ substrate. In principle, it should be possible to extract the dielectric constant $\epsilon_{G}$ of the graphene sheet. We numerically solved the Poisson equation and found that $\partial C / \partial z$ has a very weak dependence on $\epsilon_{G}$ when $\epsilon_{G} \gtrsim 2$. As a result, estimating the dielectric constant of graphene is difficult.

${ }^{12}$ Note that $\chi^{\text {no graphene }}$ varies slightly as humidity is changed. The size and the sign of the variation are consistent with previous work on $\mathrm{SiO}_{2}$ surfaces contaminated with a submonolayer of carbon (Ref. 10).

${ }^{13}$ Note that the wafer was exposed to air during manipulation before these measurements, at which point water and other molecules were likely to adsorb on the graphene sheet.

${ }^{14}$ D. V. Chakarov, L. Österlund, and B. Kasemo, Langmuir 11, 1201 (1995).

${ }^{15}$ M. Luna, J. Colchero, and A. M. Baró, J. Phys. Chem. B 103, 9576 (1999).

${ }^{16}$ W. Kim, A. Javey, O. Vermesh, Q. Wang, Y. Li, and H. Dai, Nano Lett. 3, 193 (2003).

${ }^{17}$ Y. Rudich, I. Benjamin, R. Naaman, E. Thomas, S. Trakhtenberg, and R. Ussyshkin, J. Phys. Chem. A 104, 5238 (2000).

${ }^{18}$ M. Ishigami, J. H. Chen, W. G. Cullen, M. S. Fuhrer, and E. D. Williams, Nano Lett. 7, 1643 (2007).

${ }^{19}$ E. Stolyarova, K. T. Rim, S. Ryu, J. Maultzsch, P. Kim, L. E. Brus, T. F. Heinz, M. S. Hybertsen, and G. W. Flynn, Proc. Natl. Acad. Sci. U.S.A. 104, 9209 (2007)

${ }^{20}$ J. Moser, A. Barreiro, and A. Bachtold, Appl. Phys. Lett. 91, 163513 (2007).

${ }^{21}$ J. Martin, N. Akerman, G. Ulbricht, T. Lohmann, J. H. Smet, K. von Klitzing, and A. Yacoby, Nat. Phys. 4, 144 (2008).

${ }^{22}$ S. Adam, E. H. Hwang, V. M. Galitski, and S. Das Sarma, Proc. Natl. Acad. Sci. U.S.A. 104, 18392 (2007) 